\title{
EVOLUTION OF STELLAR ACTIVITY IN EARLY POST-MAIN-SEQUENCE PHASES
}

\author{
A. MAGGIO, S. SCIORTINO \\ Istituto e Osservatorio Astronomico, Palermo, Italy
}

\section{BIANCHI}

Osservatorio Astronomico di Torino, Italy

\author{
F.R. HARNDEN JR. \\ Harvard-Smithsonian Center for Astrophysics, USA
}

R. ROSNER

Department of Astronomy and Astrophysics and Enrico Fermi Institute, University of Chicago, USA

\section{RESULTS AND DISCUSSION}

We present preliminary observational evidences on the variation of the activity level in late type stars, during the evolutionary phases on the main sequence and beyond. We have selected a sample of 51 stars (Fig. 1), lying mostly along evolutionary tracks between 1.3 and 1.7 solar masses, which have been observed in soft X-rays with the Einstein Observatory, and in UV with IUE (Maggio et al. 1990; Haisch et al. 1990). Two ROSAT targets, and four new IUE observations are also included.

We find that the radiative emission from the outer atmospheres of stars with $M>1.6 M_{\odot}$ seems to behave differently than for stars with lower mass.

On the main sequence, the X-ray luminosity of most stars with $B-V<0.42$ (spectral type F3) is relatively low, at $L_{x} \sim 3 \times 10^{28} \mathrm{erg} \mathrm{s}^{-1}$ (Fig. 2). In the early evolutionary phases beyond the main sequence, the X-ray luminosity of the higher mass stars tend to increase sistematically up to $\sim 10^{30} \mathrm{erg} \mathrm{s}^{-1}$, while the lower mass stars show an initial moderate increase followed by a drop, at $\mathrm{B}-\mathrm{V} \sim 0.6$, below our sensitivity threshold.

A mass-dependent behavior is shown also by the C IV (1550 $\AA)$ emission line strength, a transition region diagnostic. For $M>1.6 M_{\odot}$ stars near the main sequence, the C IV luminosity is $L_{C I V} \sim 10^{29} \mathrm{erg} \mathrm{s}^{-1}$. While evolution proceeds, it appears to decrease steadly, reaching our detection limit near the base of the red giant branch. On the other hand, for the $M \leq 1.6 M_{\odot}$ stars, the C IV emission shows a behavior similar to what found in $\mathrm{X}$-rays, although with a fairly large scatter in the data.

To understand the above observational picture, at least qualitatively, our working hypothesis is the following: On the main sequence, the earlier, more massive stars in our sample have too shallow convection zones for a magnetic dynamo mechanism to be efficient. The chromospheres and even the transition 
regions of these stars are dominated by mechanical heating, but not the coronae (see also Simon and Drake 1989; Simon and Landsman 1991). This explains the relatively high UV emission with respect to the X-ray emission. These stars retain an high angular momentum during the main sequence lifetime, since they are not affected by magnetic braking. While evolving, these stars develop a convection zone which triggers the onset of an efficient magnetic dynamo, which in turn provides the required coronal heating. At the same time, the mechanical heating is theoretically expected to decline (Narain and Ulmschneider 1990). On the other hand, for the lower mass stars the magnetic dynamo is already at work on the main sequence, and their rotational velocity is known to decrease with age, together with their activity level (Pallavicini et al. 1981; Maggio et al. 1987). Upon leaving the main sequence, the depth of the convection zone start to increase, but the rotation rate keeps declining, and eventually drops below some threshold value where the dynamo ceases to be effective.

To test this hypothesis we have searched for correlations between the activity level, and dynamo-related parameters, such as the rotational velocity and the Rossby number (Durney, Mihalas and Robinson 1981). The latter has been computed by properly estimating the convective turnover time from theoretical evolutionary models (Rucinsky and Vandenberg 1986).

We find that the $\mathrm{X}$-ray luminosity is not correlated with the rotation rate for the whole sample. A significant correlation is found excluding the $M>1.6 M_{\odot}$ stars on the main sequence, the $M \leq 1.6 M_{\odot}$ more evolved stars with $L_{x}<10^{28.5}$ erg $s^{-1}$, and also the most intense X-ray source (the reddest high-mass star in Fig.2). According to our hypothesis, the first two classes do not correlate because the dynamo mechanism is not effective, while for the latter object the influence of the increased convection zone depth on the efficency of the $\alpha-\omega$ dynamo cannot be neglected. In fact, a significant correlation is found between the $\mathrm{X}$-ray luminosity and the Rossby number, by excluding only the sources with the lowest X-ray emission level (Fig. 3).

\section{REFERENCES}

Durney, B.R., Mihalas, D., and Robinson, R.D. 1982, PASP, 93, 537.

Haisch, B.M., Bookbinder, J., Maggio, A., Vaiana, G.S., and Bennett, J.O. 1990, ApJ, 361, 570 .

Maggio, A., et al. 1987, ApJ, 315, 687.

Maggio, A., Vaiana, G.S., Haisch, B.M., Stern, R.A., Bookbinder, J., Harnden, F.R., Jr., and Rosner, R. 1990, ApJ, 348, 253.

Narain, U., and Ulmschneider, P. 1990, Space Sci.Rev., 54, 377.

Pallavicini, R., Golub, L., Rosner, R., Vaiana, G.S., Ayres, T., Linsky, J.L. 1981, ApJ, 248, 279.

Rucinsky, S.M., and Vandenberg, D.A. 1986, PASP, 98, 669.

Simon, T., and Drake, S.A. 1989, ApJ, 346, 303.

Simon, T., and Landsman, W. 1991, $A p J, 380,200$.

Vandenberg, D.A. 1985, ApJS, 58, 711. 


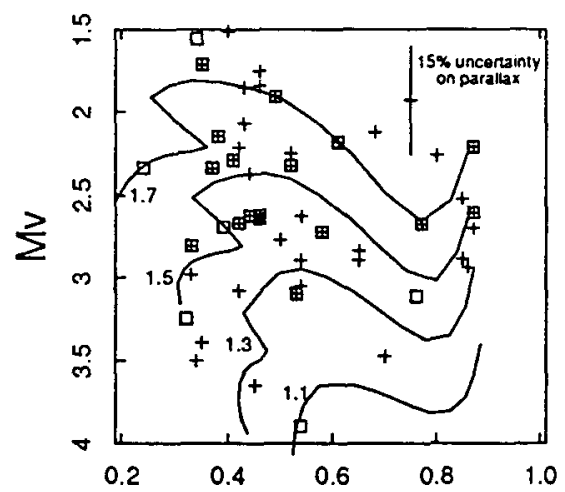

$B-V$
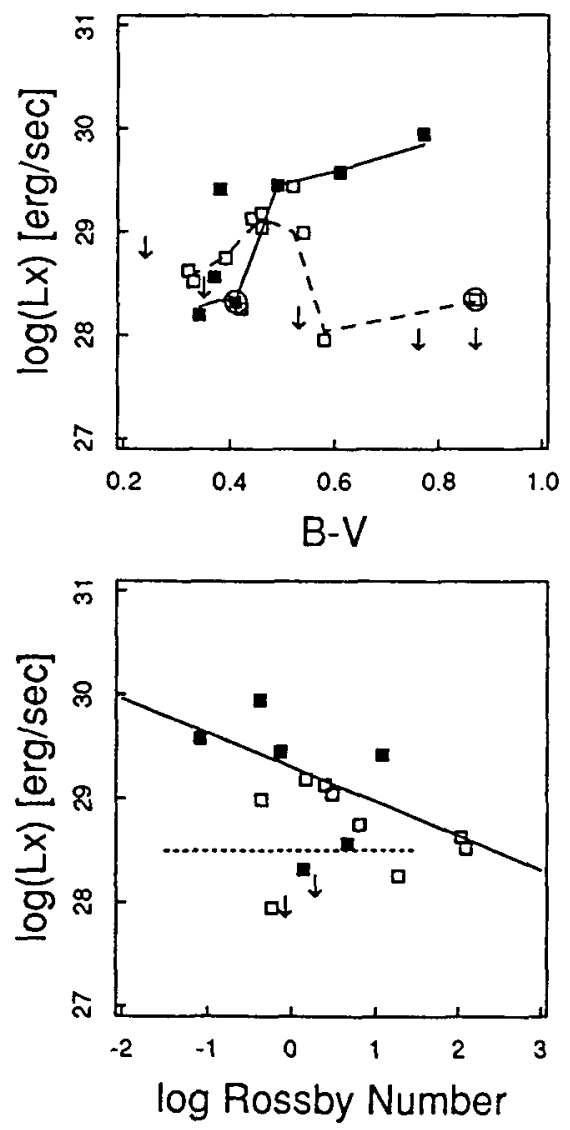

Fig. 1. H-R diagram for the stars in the selected sample. Only stars within a distance of $50 \mathrm{pc}$, and B-V color less than 0.9 have been included. Typical uncertainty on the absolute magnitude, due to an error of $15 \%$ on the parallax is also shown. Squares indicate stars observed in X-rays, while crosses are used for IUE observations. The theoretical evolutionary tracks are from Vandenberg (1985), and refer to stars with masses in the range $1.1-1.7 M_{\odot}$. These tracks have been used to estimate the star mass by interpolation. Given the uncertainty on the parallaxes, this estimate may be in error by about $\pm 0.2 M_{\odot}$.

Fig. 2. Scatter plots of X-ray luminosity vs. B-V color. Squares are used for detections, and arrows for upper limits; filled symbols identify $M>1.6 M_{\odot}$ stars; circles indicate the new ROSAT observations. The lines are the result of a robust locally weighted regression, which takes into account both detections and upper limits, applied separately to the high-mass (solid line) and low-mass (dashed line) subsamples.

Fig. 3. X-ray emission vs. Rossby number. The symbols are as in Fig. 2. The best fit power law for all stars with $\log \left(L_{x}\right)>28.5$ erg s$^{-1}$ (those below the dotted segment have been excluded), irrespective of their mass, yields a slope of $-0.34_{-0.06}^{+0.06}$ with a correlation coefficient of $-0.74_{-0.16}^{+0.10}$. We judge the fit quality reasonably good, given the uncertainties in the evaluation of the Rossby number. 